\title{
Experimental Comparison of Dynamic Tracking Performance of iGPS and Laser Tracker
}

\author{
Wang Zheng ${ }^{1}$, Mastrogiacomo Luca ${ }^{2}$, Maropoulos Paul ${ }^{1}$, Franceschini Fiorenzo ${ }^{2}$ \\ ${ }^{1}$ University of Bath - Dept. of Mechanical Engineering, Bath, BA2 7AY, United Kingdom; \\ ${ }^{2}$ Politecnico di Torino - DISPEA, Corso Duca degli Abruzzi 24, 10129 - Torino, Italy.
}

\begin{abstract}
External metrology systems are increasingly being integrated with traditional industrial articulated robots, especially in the aerospace industries, to improve their absolute accuracy for precision operations such as drilling, machining, and jigless assembly. While currently most of the metrology assisted robotics control systems are limited in their position update rate, such that the robot has to be stopped in order to receive a metrology coordinate update, some recent efforts are addressed toward controlling robots using real-time metrology data. The indoor GPS is one of the metrology systems that may be used to provide real-time 6DOF data to a robot controller. Even if there is a noteworthy literature dealing with the evaluation of iGPS performance, there is, however, a lack of literature on how well the iGPS performs under dynamic conditions. This paper presents an experimental evaluation of the dynamic measurement performance of the iGPS, tracking the trajectories of an industrial robot. The same experiment is also repeated using a laser tracker for reference. Beside the experiments results presented, this paper also proposes a novel method for dynamic repeatability comparisons of tracking instruments.
\end{abstract}

Keywords: Indoor GPS, laser tracker, dynamic performance.

\section{Introduction}

The accurate tracking of moving objects is a complex problem. It has wide applications in industry, including robot guidance, motion analysis and machine tool calibration. Dynamic tracking measurements are typically conducted using laser trackers or photogrammetry [1, 2, 3, 4 and 5]. While these systems have excellent performance, they are usually restricted by the number of points that can be measured simultaneously (laser tracker), and their volume of measurement (laser tracker and photogrammetry). Additional systems must be purchased in order to increase the coverage in volume and/or number of points, at very high cost.

The indoor GPS is a distributed metrology system that offers a relatively lower cost alternative for measuring and tracking an unlimited number of coordinates in a large volume. While the static coordinate measurement performance of the iGPS metrology system has recently been analysed in a fair amount of detail [6, 7, 8, and 9], no existing literature delved into its dynamic performance. For applications such as real-time robot guidance and the analysis of parts in motion, the dynamic tracking performance of the iGPS needed to be studied. The experiments described in this paper attempts to study and analyze the dynamic repeatability [10] measurement capabilities of the iGPS, comparing them with the performances of a laser tracker under the same experimental conditions. It has to be pointed out that, although there has been a recent new release of the iGPS software, all the results presented in this paper have been obtained using the instrument management software available at the time of the experiments. 


\section{Equipment Description}

\section{1 iGPS Technology and operating features}

Typically, the system components of iGPSTM are two or more transmitters, a control centre and a number of wired/wireless sensors (see Figure 1).

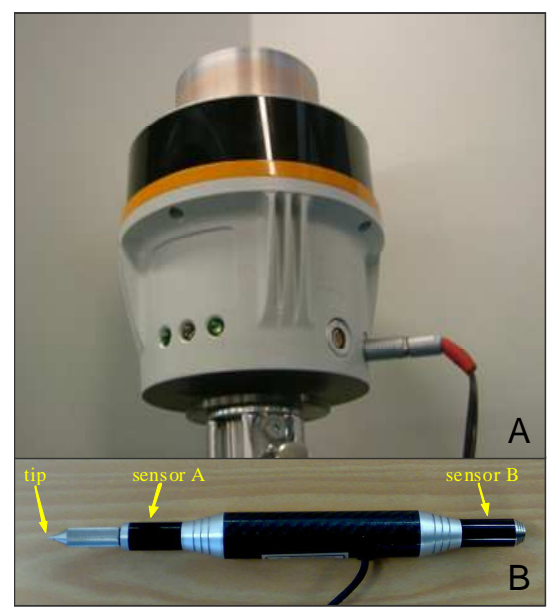

Figure 1 - A) iGPS transmitter. B) Two sensor vector bar

Transmitters operate as reference points (with known position) continually generating three signals: two infrared laser fanned beams rotating in the head of the transmitter and an infrared (IR) LED strobe. Sensors are passive elements, which can be placed on the surface of the object to be measured to receive the transmitters' signals. Before starting measurements, the locations of transmitters are solved by measuring a number of points inside the working volume, and applying scaling information between the points.

During measurements, the position $(x, y, z)$ of each sensor is calculated. Each transmitter-sensor pair acts in the same way as a Theodolite-target pair, producing two angular measurements: the horizontal (azimuth, $\varphi$ ) and the vertical (elevation, $\theta$ ) angles. Sensors can triangulate their position whenever they are located in the line-of-sight of two or more transmitters [11].

The technique used by each transmitter-receiver pair to determine the azimuth and elevation angles is as follows [11]. Each transmitter generates two rotating infrared laser beams and an infrared LED strobe. These optical signals are converted into timing pulses through the use of a photo detector. The rotation speed of the spinning head in each transmitter is set to a different value in order to differentiate between the transmitters. Additionally, the transmitter angular velocity is continuously tracked and used to convert the timing intervals into angles. As shown in the Figure 2, the two fanned beams, radiated from the rotating head of each transmitter, are tilted with respect to the rotation axis, nominally at $-30^{\circ}$ and $+30^{\circ}$. The tilt of the laser fans facilitates the calculation of the elevation angle by:

- Knowing the angles of the fanned beams $(\phi)$ which is a factory specification (see Figure 2);

- Determining the difference in timing between the arrival of laser 1 and laser 2 to the sensor $\left(t_{3}-t_{2}\right)$;

- Knowing the speed of rotation of the transmitter $(s \square 3000 \mathrm{rpm})$. 


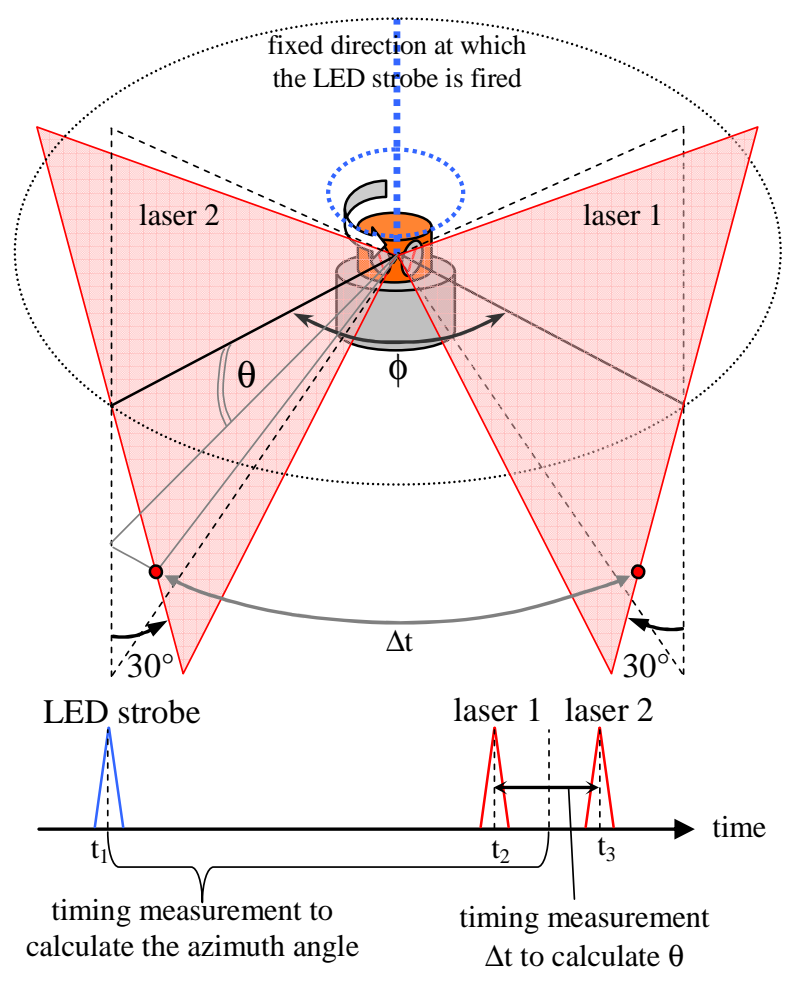

Figure 2 - Transmitter laser fans and timing diagram of the signal received by the sensor (not to scale)

The measurement of azimuth angle $(\varphi)$ requires a horizontal index, which is created by firing an omnidirectional LED strobe at a fixed direction in the rotation of the transmitter's head. Referencing the timing diagram at the bottom of the figure, the azimuth angle is determined by:

- Knowing the angles the fanned beams;

- Making a timing measurement between the strobe and the laser pulses $(\Delta t)$;

- Knowing the speed of rotation of the transmitter.

The iGPS can be used to perform either static or dynamic measurements. However, due to the positioning method used, it is believed that degradation in accuracy will occur under dynamic conditions. The transmitters' sampling rate depends on the angular speed of their rotating heads. As explained above, the spinning speed is also the unique 'ID' for each transmitter. Given that the rotation speed is around $3000 \mathrm{rpm}$, each transmitter will be able to communicate with sensors at approximately $50 \mathrm{~Hz}$. Even though differences in the transmitters sampling rate are small, it is impossible to receive concurrent data from all transmitters. The inevitable difference in data streaming is in the range of a few hundredths of a second. This effect does not create any problem for static measurements, but it will affect dynamic measurement. Figure 3 shows such a scenario, in which a sensor is moving in time $(\mathrm{t})$. The position of the moving sensor is calculated by triangulating data collected in quick secession, but while the receiver is moving. 


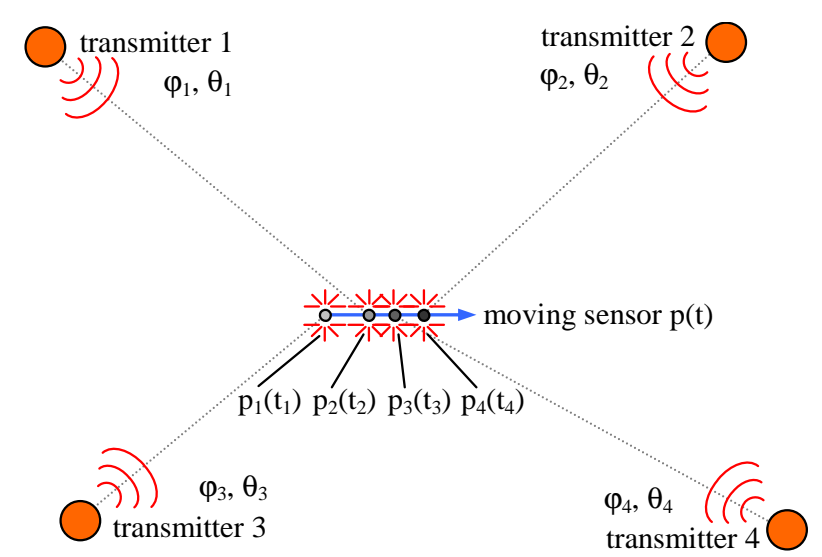

Figure 3 - If the sensor is moving, pulses from transmitters are received in different instances in time and space.

It can be assumed for the purpose of discussion that the data collection occurs by sensing information received firstly by transmitter-1, secondly by transmitter- 2 , thirdly by transmitter- 3 and finally by transmitter- 4 . At time $t_{1}$, a moving sensor's angles to the corresponding transmitter are read when it is located in position $\mathrm{p}_{1}$, at time $t_{2}$, when it is in position $\mathrm{p}_{2}$ and so on. Even if the difference consists of a few hundredths of a second, because the angles no longer resolve to correct position, location errors are produced. It is hypothesised that the faster the sensor moves, the larger this dynamic error likely will be.

\subsection{Laser Tracker}

The Laser Tracker utilises interferometry for measuring length and a pair of high resolution angle encoders to measure the horizontal and vertical angles of the laser beam. Figure 4 shows a schematic of the internal components of a typical laser tracker. In the interferometry technique a coherent laser beam of known wavelength passes through a beam-splitter. One beam is reflected back within the system while the other is aimed at a Spherical Mirror Reflector (SMR) that is a sphere with an embedded corner cubed reflector. When the two beams combine, constructive and destructive interference at the laser wavelength can be observed by the detector. The number of the bright and dark patterns is counted by the relevant electronics to calculate the distance. The SMR is used as the instrument probe, thus the laser tracker is a contact measurement system.

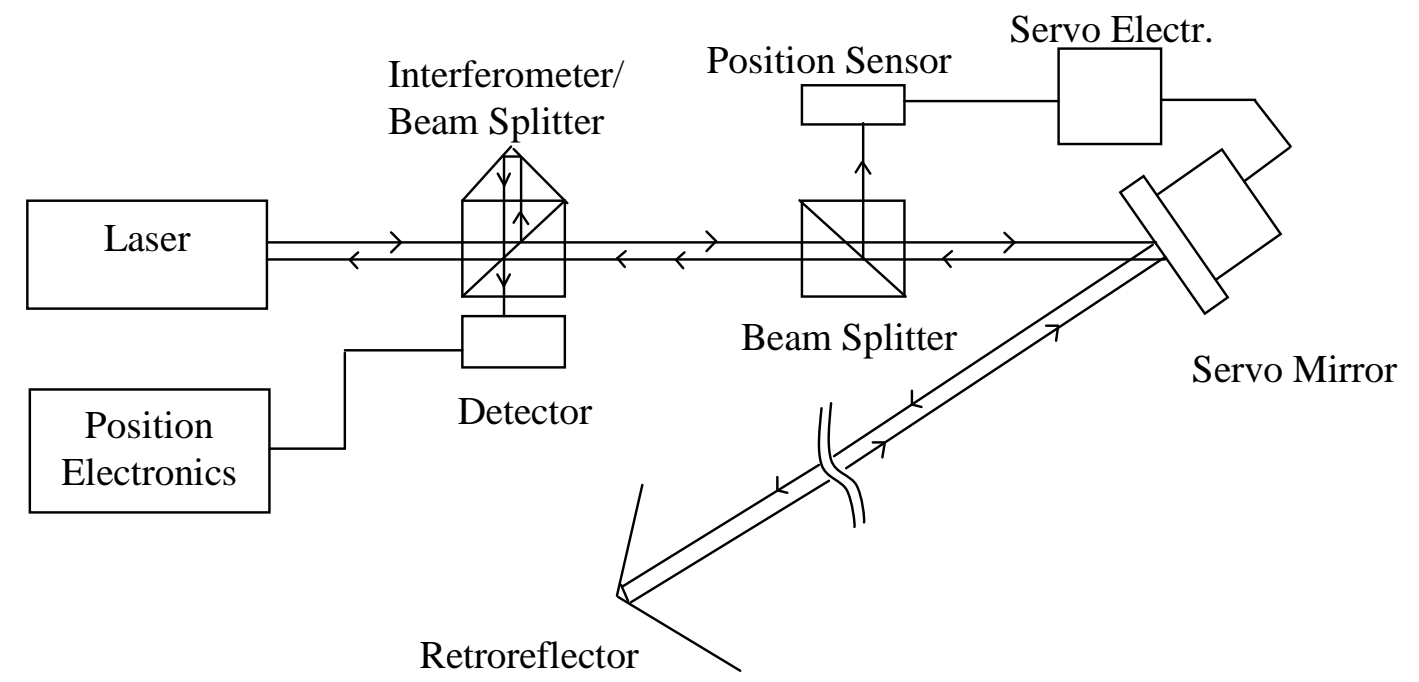

Figure 4 - Interferometry in Laser Trackers [12]. 
Laser trackers are considered to be one of the most reliable and well established metrology systems. An international standard exists for the system's performance evaluation [13]. Their main drawback is that the line of sight between the laser tracker head and the SMR must be maintained at all times, and only one SMR at any time can be tracked. Some laser trackers provide an Absolute Distance Measurement (ADM) system, which modulates the laser beam and detects the phase of the returned light [12]. By gradually reducing the modulation frequency, the absolute distance of the target can be determined with a high degree of accuracy. ADM enabled laser trackers are more user friendly, since when the line of sight is broken, the tracker can reconnect with the SMR without homing the SMR to the tracker's initial position, as is required for an interferometer system. The ease of use however, comes at the cost of a slight decrease in accuracy [14].

The FARO Tracker SI using in this experiment has a single point angular accuracy (2 sigma) of $18 \mu \mathrm{m}+3 \mu \mathrm{m} / \mathrm{m}$, and distance accuracy (2 sigma) of $20 \mu \mathrm{m}+1.1 \mu \mathrm{m} / \mathrm{m}$ in ADM mode. [14]

\subsection{Industrial Robot}

An industrial articulated robot in the laboratory was used to generate the trajectories for the experiment. The robot offered a fast and simple way of generating a number of test trajectories, and the speed of the movement can also be easily altered.

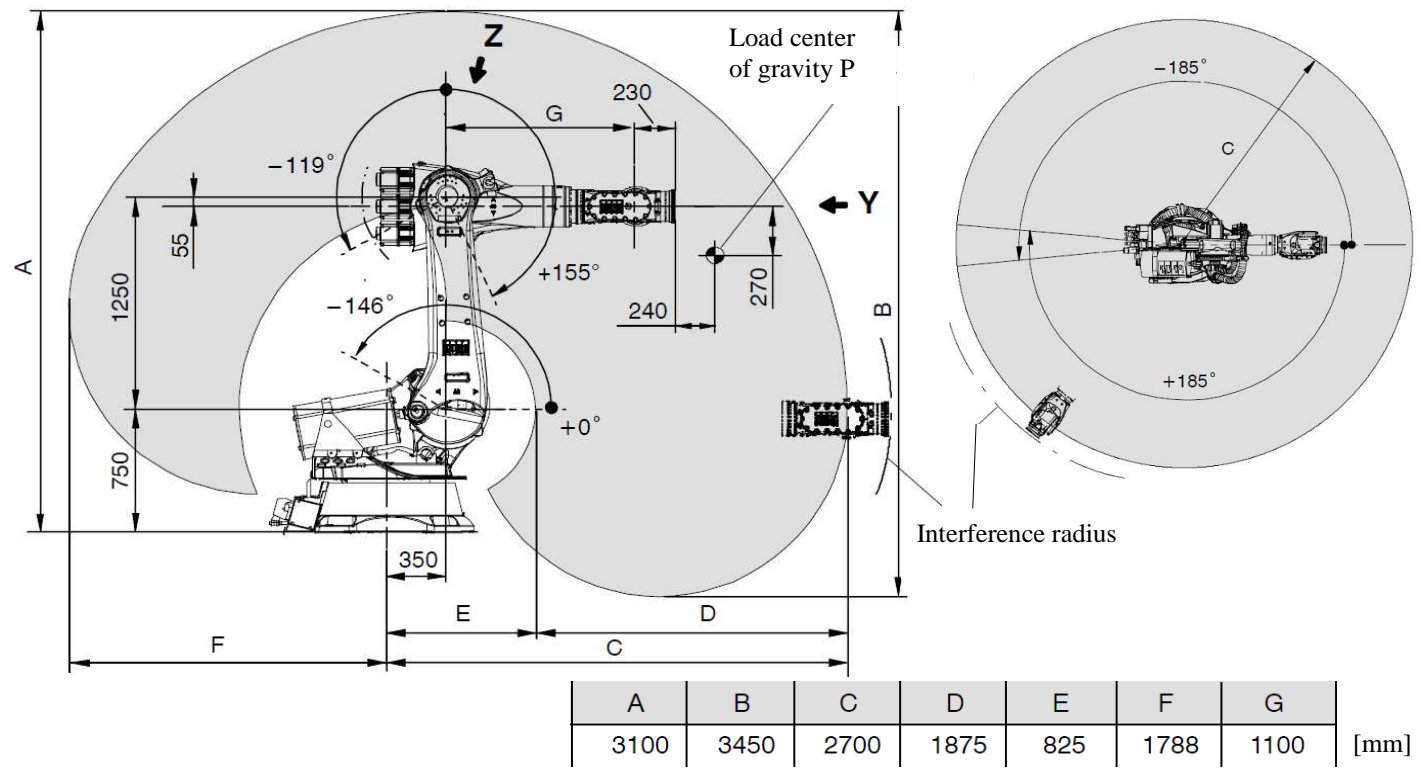

Figure 5 - Drawing of the working envelope of the KR240-2. [15]

The KUKA KR240-2 robot (Figure 5) used in this experiment has a static point repeatability of $\pm 0.12 \mathrm{~mm}$, and maximum movement speed of $2 \mathrm{~m} / \mathrm{s}$. A two hour static repeatability test conducted on the robot before the experiment using a grid of 30 points in the robot working volume confirmed that the repeatability of the robot is performing within the manufacturer's specifications [16].

\section{Equipment Setup and Experiment Procedure}

\subsection{Equipment Layout}

The measurement instruments are arranged as shown in Figure 6. The laser tracker is placed approximately 4 metres from the robot base. The iGPS transmitters are arranged in a ' $\mathrm{C}$ ' shaped network approximately 6-7 metres from the robot base. The lengths of 
the robot trajectories are approximately $1 \mathrm{~m}, 1.7 \mathrm{~m}$ and $0.75 \mathrm{~m}$ for $\mathrm{X}, \mathrm{Y}$ and $\mathrm{Z}$ respectively (see Figure 6).

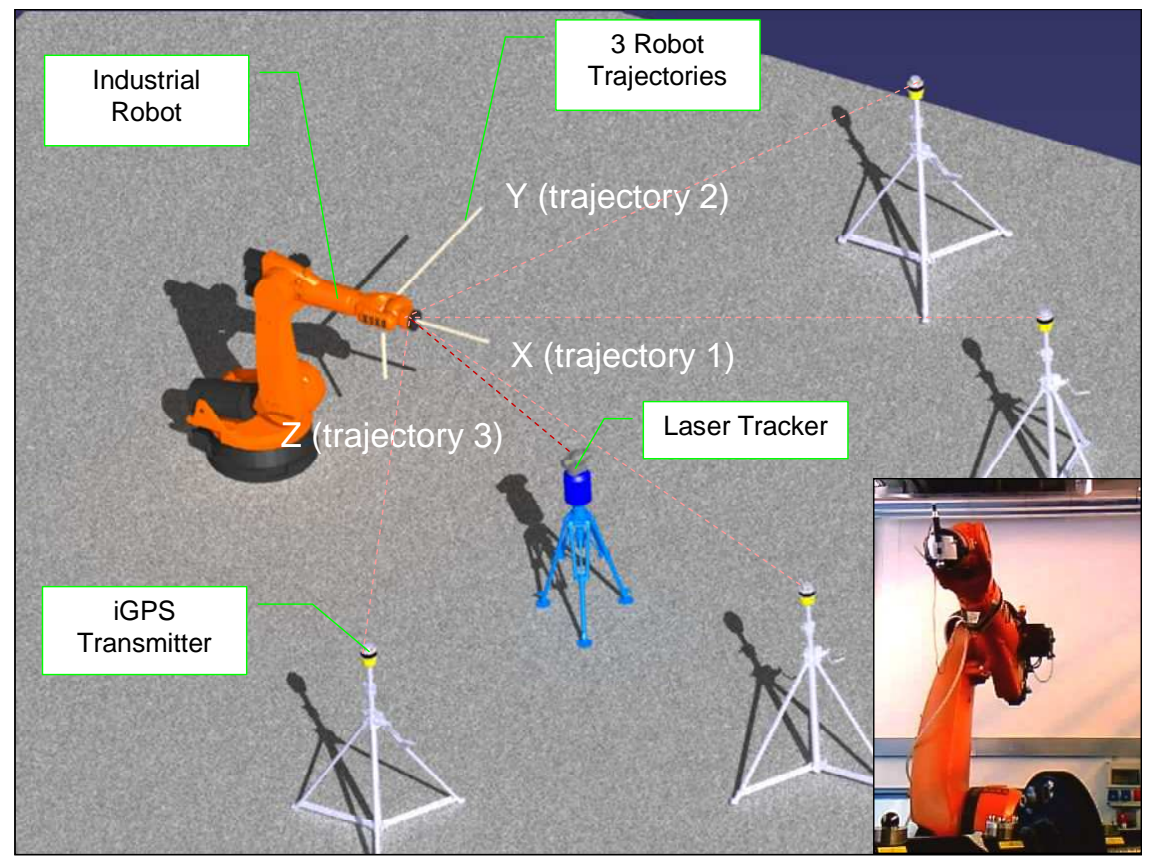

Figure 6 - Approximate experiment layout illustration, and picture of the robot carrying the iGPS vector bar

The iGPS transmitters' positions in the network were calculate through a bundling, procedure. For this experiment, 8 points in the working volume are measured using the vector bar, which provided enough angular and scale information for the software to solve for the transmitter positions. A more accurate scale bar was then setup using a two metres carbon fibre artefact from the National Physical Laboratory, which was measured using the laser tracker in interferometry mode. The measurement of the scale bar obtained using the iGPS was compare to that from the tracker, the ratio of the two measurements was used to rescale the iGPS network.

A 1.5 inch laser tracker SMR nest was attached to the end of the robot arm. The location of this nest is measured using a 1.5 inch SMR with the laser tracker, and the iGPS vector bar with a 1.5 inch tip probe (see Figure 6). The centre of the SMR was defined as the Tool Centre Point (TCP) of the robot.

Within the measurement range in the experiment, the laser tracker is expected to have a single point measurement uncertainty of $30-40 \mu \mathrm{m}$ (2 sigma) according to the manufacturer's specifications [14], and for similar sized network setups, the iGPS has been demonstrated to be capable of single point static uncertainties of $500-1000 \mu \mathrm{m}(2$ sigma) [8]. While the static repeatability of the robot have been shown to be less than $110 \mu \mathrm{m}[16]$, its dynamic performance is unclear.

\subsection{Experiment Procedure}

The industrial robot was programmed to repeatedly run through three mutually orthogonal linear trajectories (see Figure 6). Measurement data were collected at six robot movement speeds ranging from $1 \mathrm{~cm} / \mathrm{s}$ to $1 \mathrm{~m} / \mathrm{s}$. In every program loop, each of the three trajectories was run twice, once forwards and once backwards. The program is looped 4-6 times at each robot speed. Reference data sets for each instrument were constructed from the slowest speed trials, which is then used as a basis for comparison 
with higher speed trials, such that the two instruments are not directly compared with each other, but to the reference data constructed by their own slowest runs.

A total of 64 sets of data were collected from the two instruments at six different speeds $(1 \%, 10 \%, 30 \%, 50 \%, 75 \%$ and $100 \%$ of $1 \mathrm{~m} / \mathrm{s})$ in three axis and two directions. The $\mathrm{Z}$ trajectory data was not available for $75 \%$ and $100 \%$ speeds, due to robot joint speed limitations.

\section{Results}

As an example, recorded data from one of the linear robot trajectories ( $\mathrm{Y}$ direction) at $1 \mathrm{~m} / \mathrm{s}$ speed is shown in Figure 7. The figure highlights the effect of movement on the position of the Vector Bar as perceived by the iGPS software compared to the laser tracker data. At the end points of the trajectory, where speed is zero, the iGPS measurements are very close to that of the laser tracker. However, as the robot accelerates to the specified trajectory speed, clear biases are introduced into the iGPS readings. The direction of the biases appears to depend on the direction of the robot movement.

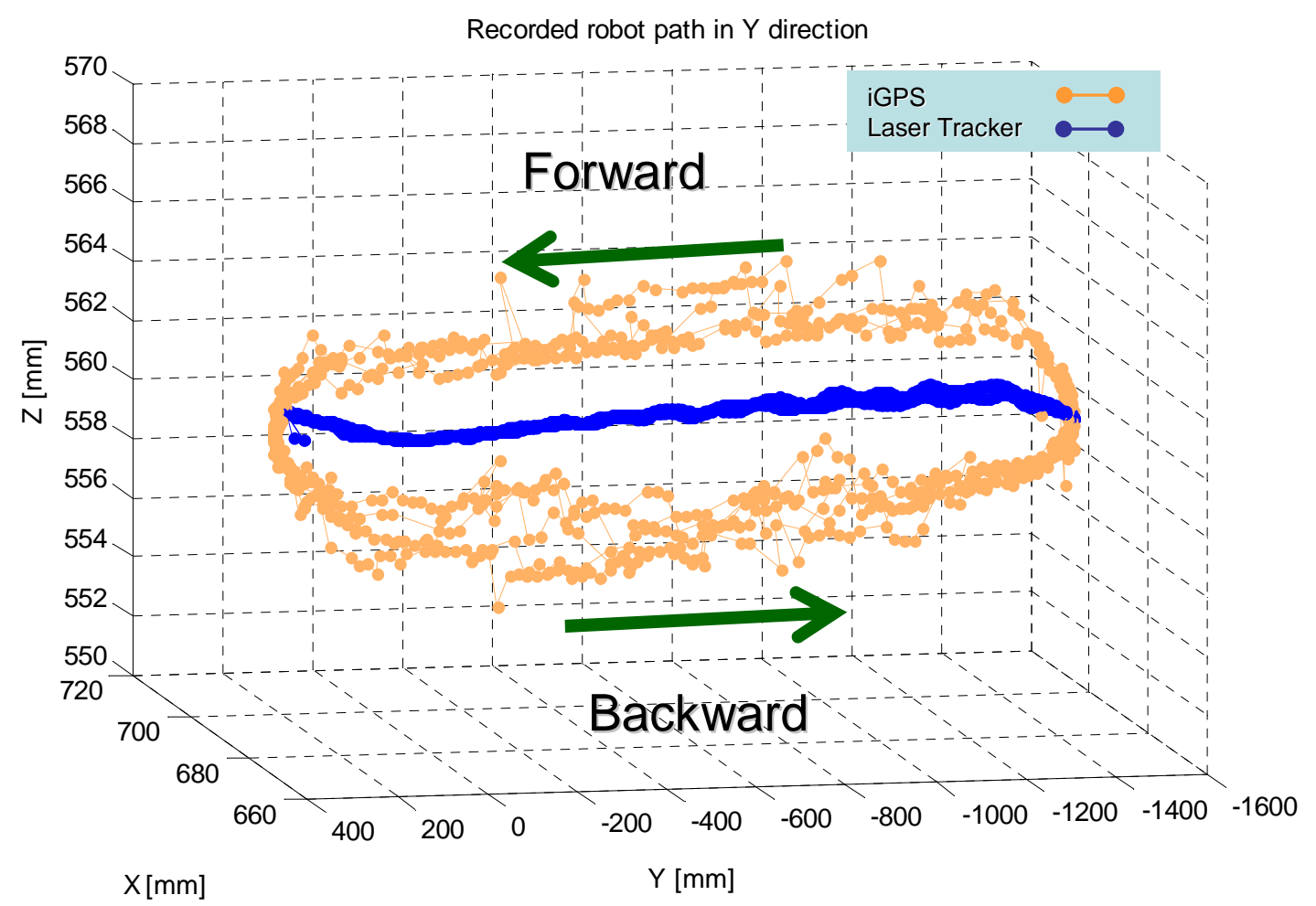

Figure 7 - Difference between iGPS and laser tracker measurements at 1m/s.

Please note Figure 7 is only included to illustrate the iGPS bias generated as a result of movement, not to make any statement about the relative accuracies of the iGPS compared with the tracker. Comparisons of accuracies require more rigorous methods of fitting to convert one instrument's coordinate system into the other. Section 4.1 and 4.2 propose an approach to this problem. First a common coordinate reference system is defined using the tracking data recorded at the lowest speed. Then the tracking results are compared and visualized projecting them on a common reference plane. 


\subsection{Reference Line Generation}

In order to generate a reference data set to enable the comparison between data recorded at different speeds, a line in 3D space is fitted to the data collected over 4-6 runs at the slowest speed $(1 \mathrm{~cm} / \mathrm{s})$ for each robot trajectory. The Robust Least Squares (RLS) fitting method was used to reduce the influence of outlier points on the fitting result [17].
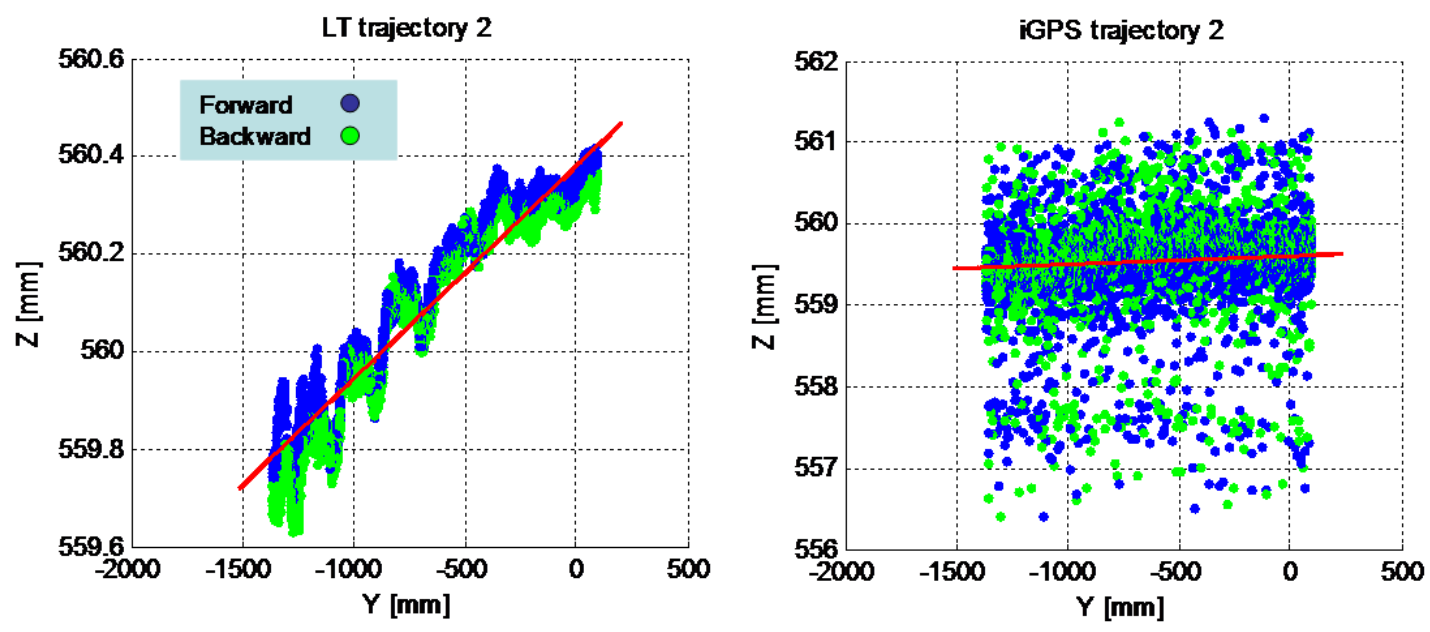

Figure 8 - Robust Least Squares (RLS) fitting of reference line. The first and last 150mm of data from each trajectory are deleted.

Figure 8 shows the result of the fitting for the Y trajectory. Since the robot took $100 \mathrm{~mm}$ to accelerate to a constant linear speed at the TCP, the first and last $150 \mathrm{~mm}$ of the trajectory were deleted before the data is further processed. This ensures that only the constant speed portions of the trajectories were compared to each other.

\subsection{Results Analysis}

Due to the difficulties in quantitatively and qualitatively comparing large amounts of 3D point cloud data, a method is developed to visualize the differences in the recorded data. Using the 3D reference line generated in 4.2 as the normal vector of a plane, it is possible to project the trajectory measurements on this plane. The coordinate system of the trajectory data is transformed into that of the reference line. The resulting 2D plot represents a view of the data along the reference line, as shown in Figure 9. Also included in the figure are standard deviation ellipses to aid visualization of the grouping of data for different speeds and directions of travel. In this figure, the colors differentiate the direction of travel and movement speed. 


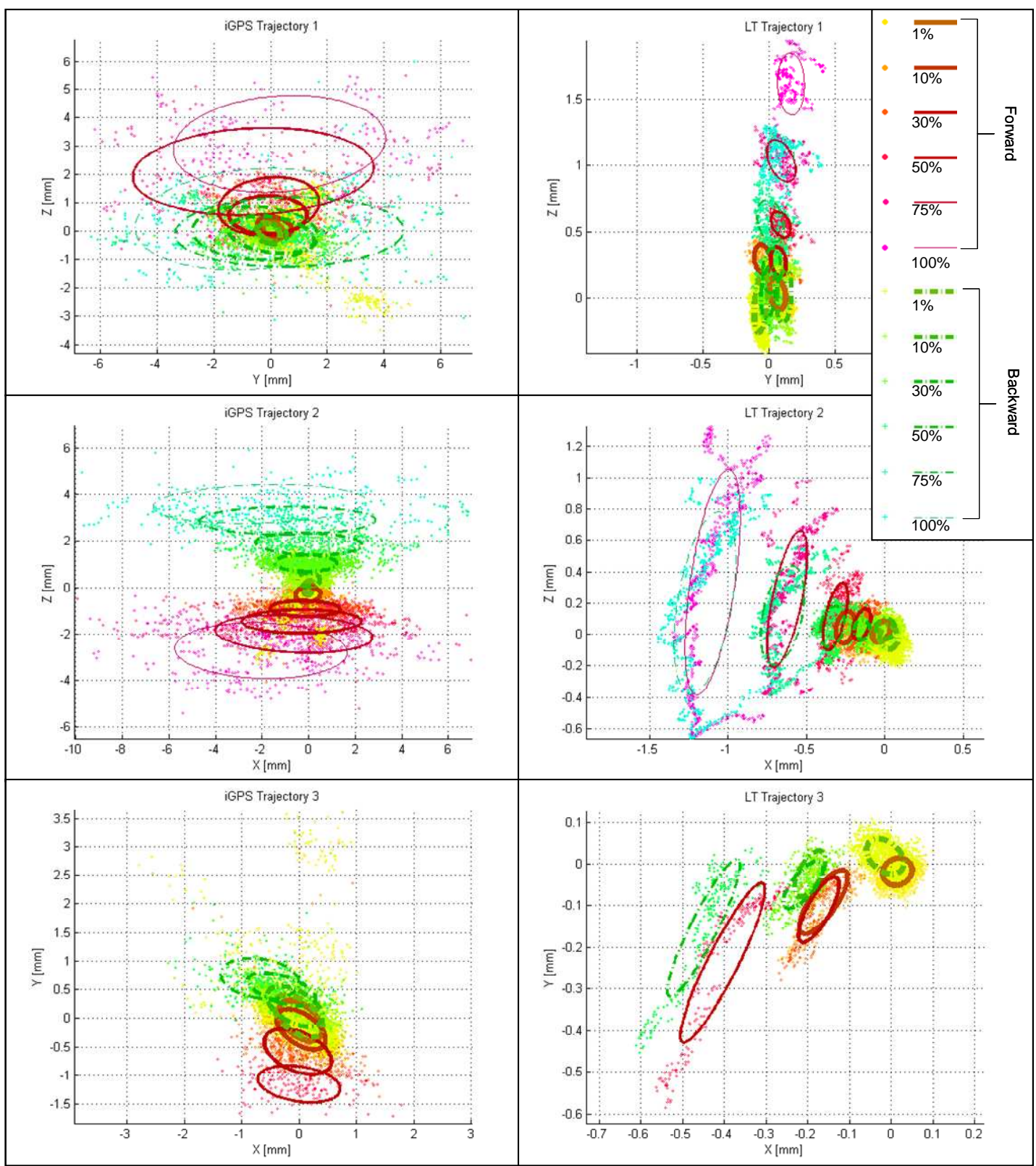

Figure 9 - Projected three trajectory measurements by the iGPS and laser tracker, including standard deviation ellipses. Percentages shown in the legend are robot speeds as a percentage of $1 \mathrm{~m} / \mathrm{s}$.

A clear difference between the robot trajectory as measured by the laser tracker and the iGPS is the overall scatter of the data points. The distribution of the iGPS data is much wider than the laser tracker, with more outliers. It can also be seen that the iGPS appears to develop somewhat symmetrical biases about the reference line for opposite directions of travel. This effect is much less visible in the laser tracker data, in which the robot movement error is the predominant source of error, which increases with robot speed, but is fairly repeatable irrespective of the directions of travel.

The distribution of the distance of each measured point to the reference line at different robot speeds can be studied quantitatively by plotting their mean and distribution against speed, as shown in Figure 10. 

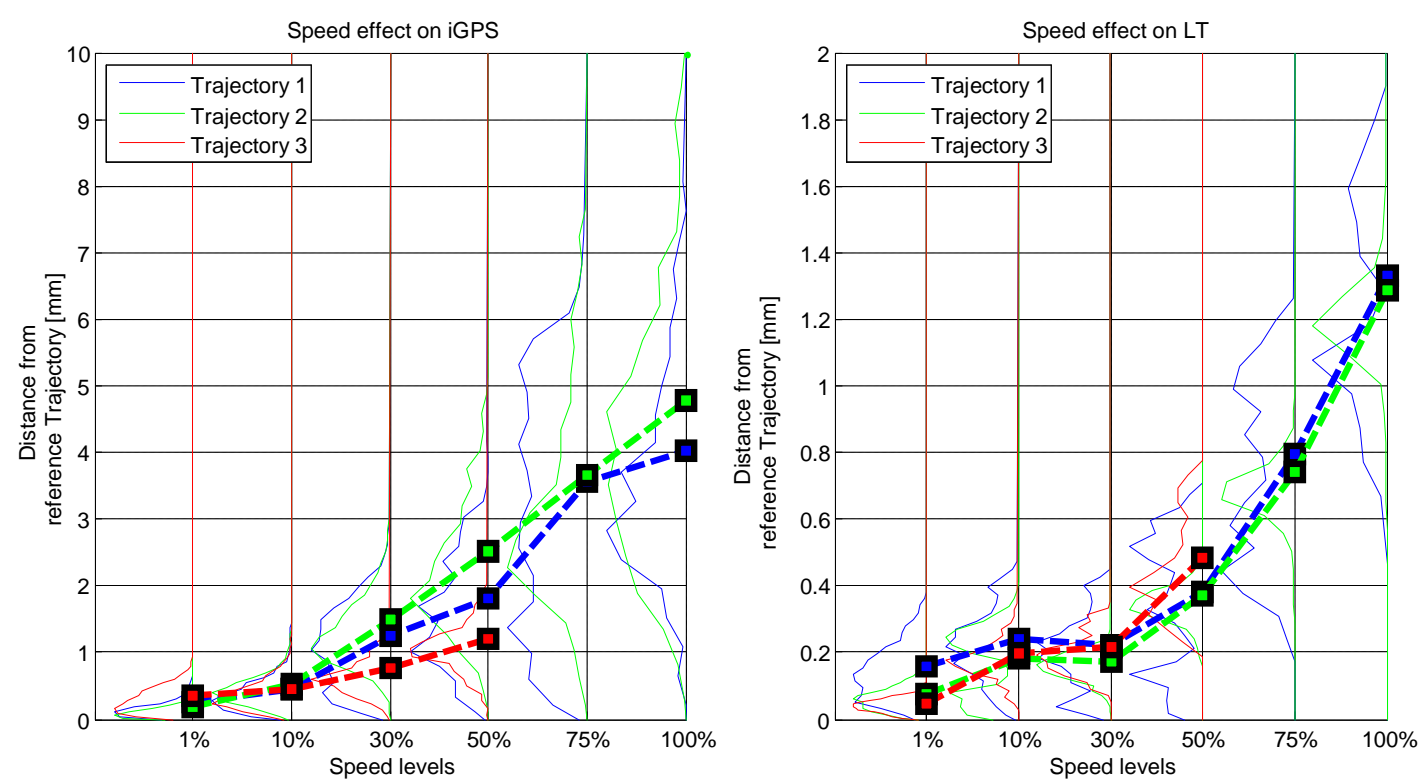

Figure 10 - Mean and distribution of the distance from the recorded coordinate data to the theoretical linear trajectories for iGPS and laser tracker. Percentages shown are robot speeds as a percentage of $1 \mathrm{~m} / \mathrm{s}$.

As speed is increased, the accuracy of the robot, iGPS and laser tracker are degraded. It appears that at least up to $10 \mathrm{~cm} / \mathrm{s}$, the iGPS is capable of providing tracking data affected by reasonably low variability and small bias. The iGPS is clearly more affected by higher robot speed, with distances from the reference trajectory up to $4-5 \mathrm{~mm}$ at $1 \mathrm{~m} / \mathrm{s}$ compared to the $1.2-1.4 \mathrm{~mm}$ of the laser tracker. The reason of this bias can be attributed to both the tracking instruments and robot movement inaccuracy.

If the robot paths are assumed to be repeatable between the laser tracker and iGPS trials, the amount of bias error introduced when the iGPS sensor is moving at $1 \mathrm{~m} / \mathrm{s}$ is expected to be at least $3-4 \mathrm{~mm}$ on average.

On the contrary it is difficult to state something about laser tracker performances in dynamic conditions since we do not have any reference for a comparison, but given that the tracker accuracy is estimated to be in the range of 30-40 $\mu \mathrm{m}$ [14], the tracker bias shown in Figure 10 is likely dominated by the error of the robot.

Considering the distribution of the distances from the reference trajectory, for both the laser tracker and the iGPS it is possible to notice a slight bimodal distribution due to the forward/ backward effect of robot movement discussed above. It is also evident how the data variance increases as the speed grows.

Although in general the repeatability of the measurements degrades as speed is increased, it can be said that iGPS and laser tracker have comparable repeatability performances up to speeds of $10 \mathrm{~cm} / \mathrm{s}$. Then while the laser tracker keeps performing with a bias smaller than $1 \mathrm{~mm}$ until $75 \mathrm{~cm} / \mathrm{s}$, the iGPS performances quickly reach relatively high level of bias.

\section{Summary and Conclusions}

In this paper, the experimental results of the dynamic performance tests of the iGPS metrology system are presented, and compared to the results of the same experiments conducted on a laser tracker. As expected the experiments showed that, a bias error is introduced to an iGPS sensor while it is moving at speed. The direction and magnitude of this bias appears to depend on the movement direction and speed. Further, it was determined that for speeds below $10 \mathrm{~cm} / \mathrm{s}$, the iGPS is capable of producing relatively 
repeatable tracking data. However, as speed is increased, the tracking accuracy degrades. At $1 \mathrm{~m} / \mathrm{s}$, the mean tracking error can be on the order of $3-4 \mathrm{~mm}$.

The aim of this paper was not to make a judgement on the iGPS dynamic performances compared to that of laser tracker. Both of the metrology instruments are normally used in static conditions but also in dynamic contexts, generally to track objects during assembly processes. The experiments conducted showed that for speed lower than $10 \mathrm{~cm} / \mathrm{s}$, which is a relatively high speed in assembly, iGPS and laser tracker repeatability performances are similar. Since the aim of assembly is that of matching two or more components there is a need for tracking six or more points (at least three for each component). While this can be done using just one iGPS network, performing the same task using six laser trackers may be prohibitively expensive.

Even if laser tracker static and dynamic accuracies are generally better, the iGPS can be more convenient than laser tracker depending on the function and the speed of the points to be tracked. Furthermore at the time of the writing of this paper, the developers of the iGPS have informed the authors that a new software system that may reduce some of the dynamic bias errors has been released. Further research efforts can be addressed in the direction of evaluating the improvements introduced by the new release of the iGPS software in both the static and dynamic performance of the instrument.

\section{References}

1. Amir Kayani, Jafar Jamshidi. "MEASUREMENT ASSISTED ASSEMBLY FOR LARGE VOLUME AIRCRAFT WING STRUCTURES”. Proceedings of DET2007, 4th International Conference on Digital Enterprise Technology, Bath, (2007).

2. Brian Rooks. "Automatic wing box assembly developments". Industrial Robot, Volume 28, Number 4, Pages 297-301 (2001).

3. S J Eastwood, $\mathrm{P}$ Webb, and C Mckeown. "The use of the $T^{2}$ manufacturing system on a double-curvature aerospace panel". Proceedings of Institute of Mechanical Engineers Vol. 217, Part B: J. Engineering Manufacture, page 849, (2003).

4. P Webb, S J Eastwood. "An evaluation of the $T I^{2}$ manufacturing system for the machining of airframe subassemblies. Proceedings of Institute of Mechanical Engineers Vol. 218 Part B: J. Engineering Manufacture, page 819, (2004).

5. Giirsel Alici, Bijan Shirinzadeh. "Laser Interferometry Based Robot Position Error Modelling for Kinematic Calibration" Proceedings of the 2003 IEEE/RSJ, Intl. Conference on Intelligent Robots and Systems, Las Vegas, Nevada October (2003)

6. Maisano Domenico; Jafar Jamshidi; Fiorenzo Franceschini; Paul G Maropoulos; Luca Mastrogiacomo; Antony R Mileham; Geraint W Owen, "INDOOR GPS: SYSTEM FUNCTIONALITY AND INITIAL PERFORMANCE EVALUATION, INTERNATIONAL JOURNAL OF MANUFACTURING RESEARCH”, pp. 335349, 2008, Vol. 3, n. 3 (2008)

7. J. E. Muelaner, Z. Wang, J. Jamshidi, P. G. Maropoulos, A R Mileham, E. B. Hughes, and A. B. Forbes. "iGPS - AN INITIAL ASSESSMENT OF TECHNICAL AND DEPLOYMENT CAPABILITY" Proceedings of the 3rd International Conference on Manufacturing Engineering (ICMEN), Chalkidiki, Greece (2008).

8. Z. Wang, J. Jamshidi, P. Maropoulos, G. Owen, and T. Mileham. "EXPERIMENTAL DEPLOYMENT OF THE INDOOR GPS LARGE VOLUME METROLOGY SYSTEM IN A LARGE SCALE PRODUCTION FACILITY" Proceedings of the 3rd International Conference on Manufacturing Engineering (ICMEN), Chalkidiki, Greece (2008).

9. Jody Muelaner, Ben Hughes, Alistair Forbes, Paul Maropoulos, Jafar Jamshidi, Zheng Wang, and Wenjuan Sun. "iGPS capability assessment" Large Volume Metrology Conference, Liverpool (2008) 
10. VIM "International Vocabulary of Basic and General Terms in Metrology, Third Edition". ISO/DG 99999 (Geneva, Switzerland: International Organization for Standardization) (2004).

11. ARCSecond. "Indoor GPS technology for Metrology", White Paper 071502, Dulles, Virginia: ARCSecond (2002)

12. Estler WT, Edmundson KL, Peggs GN and Parker DH, "Large Scale Metrology-An Update", CIRP Annals, NIST Technipubs (2002)

13. ASME B89.4.19. "Performance Evaluation of Laser-Based Spherical Coordinate Measurement Systems" (2006)

14. FARO EUROPE GmbH \& Co. KG. "New Faro Laser Tracker SI. 2: Tougher with Exclusive Features" Faro UK technical specification sheet (2004)

15. KUKA Roboter GmbH, Germany. "KR 240-2 - KR 240 L210-2 - KR 240 L180-2 (Serie 2000) Technical Data" KWM--Nr. 841612--86/D+E/3/04.05 (2002)

16. Zheng Wang. "KUKA KR240 Robot Repeatability Study", Airbus ALCAS internal report (2008)

17. Street, J. O., R. J. Carroll, and D. Ruppert. "A Note on Computing Robust Regression Estimates via Iteratively Reweighted Least Squares." The American Statistician. Vol. 42, 1988, pp. 152-154. 